\title{
International Normalized Ratio (INR) as a Prognostic Tool in Isolated Traumatic Brain Injury (TBI)
}

\author{
Dewaraj Velayudhan ${ }^{1}$, Anmol Nagaraj ${ }^{2}$, Girish Menon ${ }^{2}$, Vijetha Shenoy Belle ${ }^{3} \&$ Asha Kamath ${ }^{4}$ \\ ${ }^{1}$ Kasturba Medical College Manipal, Manipal Academy of Higher Education Manipal, India. \\ ${ }^{2}$ Department of Neurosurgery, Kasturba Medical College Manipal, Manipal Academy of Higher Education Manipal, India. \\ ${ }^{3}$ Department of Biochemistry, Kasturba Medical College Manipal, Manipal Academy of Higher Education Manipal, India. \\ ${ }^{4}$ Department of Statistics, Prasanna School of Public Health, Manipal Academy of Higher Education, India.
}

Copyright: (02021 Dewaraj Velayudhan et al. This is an open access article distributed under the terms of the Creative Commons Attribution License, which permits unrestricted use, distribution, and reproduction in any medium, provided the original author and source are credited.

\section{ABSTRACT}

Traumatic brain injury (TBI) is the leading cause of death in trauma patients in various parts of the world including India. TBI can be further classified as primary and secondary injuries. Coagulation cascade is affected in TBI. This is called as acute traumatic coagulopathy (ATC). The severity of coagulopathy correlates with degree of primary injuries thus affecting the prognosis of patients. Since the prognosis of isolated TBI can be a challenge to predict at times, we wanted to study the potential of International Normalized Ratio (INR) test as a prognostic tool in isolated TBI. INR reflects the coagulation status and is a cheap and rapid investigation. In most trauma cases, it is a routine test as well. We collected the INR value a admission of 105 isolated TBI cases over a period of three months. Then, patients were followed-up and their outcome at three months from admission is scored using Glasgow Outcome Scale. The relationship of INR with GOS was studied. From our limited study, we found that INR of 1.06 or lesser predicts a better prognosis in cases of isolated TBI.

Keywords: Traumatic brain injury, International normalized ratio, Prognostic tool, Glasgow coma scale, Glasgow outcome scale.

\section{Introduction}

An epidemiology study in 2002 reports; nearly 1.5 million to 2 million people are injured and 1 million succumb to death every year in India [1]. By affecting physical, psychological, cognitive, emotional and social aspects adversely, traumatic brain injury is also a major cause of disability and socioeconomic losses in India. Road traffic accident is the major factor followed by falls and violence. Numbers are expected to be higher at present day. Hence, this shows the intense burden of the issue, highlighting the importance of an efficient, cost-effective prognostic tool.

Traumatic brain injury (TBI) is also known as acquired brain injury [2]. TBI can be classified in several ways. One such way is primary and secondary injuries. A primary injury is due to mechanical force and occurs instantly whereas secondary injury is not mechanically induced and it may be delayed from the moment of impact. Major mechanisms playing role in primary injury are contact and acceleration-deceleration. These in turn cause anatomical and physiological impairment. Intracranial hematoma is an example. Secondary injury on the other hand is due to decrease in cerebral blood flow as TBI ignites a viscous cascade of events, ultimately leading to neuronal death.

Many changes occur following a TBI. One that concerns our study is traumatic injury-associated coagulopathy [3]. It is also known as acute traumatic coagulopathy (ATC). This condition has been reported significantly higher in patients with TBI despite the fact there is lesser bleeding in restricted receipt of volume of fluids in isolated TBI cases. This abnormal clotting occurs in 10-20\% of head-injured patients [4]. Besides exacerbating secondary brain injury, ATC may also be a marker of the degree of the primary injury. There are several mechanisms proposed to explain acute traumatic coagulopathy in TBI. One of it is the tissue factor hypothesis. Brain tissue is rich in 
thromboplastin, and TBI activates the extrinsic pathway and leading to initial transient hypercoagulable state. This in turn leads to secondary consumptive coagulopathy resulting in a hypo-coagulable state $[5,6]$. The other proposed mechanism is explained via over-activation of protein $C$ pathway [6]. Trauma causes hypoperfusion and damages the endothelium, thus activating protein $\mathrm{C}$ pathway. Protein $\mathrm{C}$ inhibits coagulation factors; Va and VIIa by cleaving them. Another hypothesis states brain-derived microparticles (BDMP) released from an injured brain are responsible in inducing a hypercoagulable state that rapidly turns into consumptive coagulopathy [7].

International Normalized Ratio (INR) is a measure of the extrinsic pathway of coagulation, hence determining the clotting tendency of blood. INR investigation is part of the routine investigation for all trauma patients in our hospital. It is a cheap and rapid test. Since the prognosis in isolated TBI is variable and difficult to judge, we wanted to further explore the potential of INR in predicting the prognosis in isolated TBI. By doing so, INR can be a more cost-effective test.

\section{Aim and Objective}

Our aim in this study is to find the cut-off value of INR to predict good prognosis in isolated TBI patients.

\section{Methods}

A pilot prospective observational study was designed with the approval from Scientific Review Board and Ethics Committee of Kasturba Hospital, Manipal. Written informed consent was obtained from patients or their next-of-kin when not possible. It was conducted for six months from April 2015 - August 2015 involving patients admitted in Neurosurgery Department from the trauma and triage of the hospital. The first three months were spent in recruiting the data and the next three months were spent in following up the patients at three months of discharge.

A total of 105 patients from age group 18-65 years old were involved in our study. Ages below 18 years (paediatric age group) and ages more than 65 years (geriatric age group) were excluded to avoid age as a confounding factor in affecting the prognosis.

Only patients with isolated traumatic brain injury were included. These patients were identified using anatomical based Abbreviated Injury Score (AIS). There are six body regions involved in this scoring system; head or neck, face, chest, abdominal or pelvic contents, extremities or pelvic girdle and external. The scoring is as follows:

\begin{tabular}{|c|l|}
\hline AIS & Injury description \\
\hline $\mathbf{1}$ & Minor \\
\hline $\mathbf{2}$ & Moderate \\
\hline $\mathbf{3}$ & Serious \\
\hline $\mathbf{4}$ & Severe \\
\hline $\mathbf{5}$ & Critical \\
\hline $\mathbf{6}$ & Maximal/Untreatable \\
\hline
\end{tabular}


Patients with AIS score 3 or more for body regions of face, chest, abdominal or pelvic contents, extremities or pelvic girdle and external were excluded as we aim to study patients with isolated TBI only.

All those with history of coagulopathy diseases, on anti-coagulation therapy and history of liver diseases were excluded as these conditions affect the coagulation pathway.

INR value at admission was recorded.

Glasgow Outcome Scale was used to assess the progress of patients. The following is a table comprising of the scale.

\begin{tabular}{|l|l|}
\hline \multicolumn{1}{|c|}{ GOS score } & \multicolumn{1}{|c|}{ Description } \\
\hline $\mathbf{1}$ - Death & Non-survival \\
\hline $\mathbf{2}$ - Persistent vegetative state & Minimal responsiveness \\
\hline $\mathbf{3}$ - Severe disability (conscious but disabled) & Dependent on others for daily support \\
\hline $\mathbf{4}$ - Moderate disability (disabled but independent). & Can work in sheltered setting \\
\hline $\mathbf{5}$ - Good recovery & Resumption of normal life despite minor deficits \\
\hline
\end{tabular}

GOS score $1-3$ are categorized as poor outcome where $4-5$ as good outcome. The distinguishing cut-off that separates group 3 and 4 into different outcome categories is the patient's independence in carrying out daily activities of life [8].

Glasgow Coma Scale of the patients was also recorded to investigate its relationship with INR. It was divided into two groups too; high risk and non-high risk groups [8,9]. High risk group included GCS of 3-8 while the non-high risk group included GCS $9-15$. Below shows the component of GCS.

\begin{tabular}{|c|l|l|l|}
\hline Score & Best Motor Response & Best Verbal Response & Best Eye opening \\
\hline $\mathbf{1}$ & None & None & None \\
\hline $\mathbf{2}$ & Decerebrate posturing & Mutters intelligibly & Opens to pain \\
\hline $\mathbf{3}$ & Decorticate posturing & Inappropriate speech & Obeys command \\
\hline $\mathbf{4}$ & Withdraws away from pain stimulus & Confused & Opens spontaneously \\
\hline $\mathbf{5}$ & Localising response to pain & Alert and orientated & - \\
\hline $\mathbf{6}$ & Obeys command & - & - \\
\hline
\end{tabular}

Data was analysed using commercial software SPSS version 16.0. The relationship of both INR with GCS and INR with GOS is studied using Spearman's rank correlation coefficient test, a non-parametric test. The median values of INR in different groups were obtained using Mann Whitney $\mathrm{U}$ test, a non-parametric test as well. $p$-value of $<0.05$ 
was considered to be statistically significant. ROC curve was used to determine the cut-off value of INR in predicting the good outcome among isolated TBI.

\section{Results}

The 105 patients with isolated Traumatic Brain Injury have been grouped as follows:

4.1 Groups based on risk category

\begin{tabular}{|c|c|c|c|}
\hline Risk category & GCS & Number of Patients & Percentage (\%) \\
\hline Non-high & $9-15$ & 82 & 78.1 \\
\hline High & $3-8$ & 23 & 21.9 \\
\hline \multicolumn{2}{|c|}{ Total } & 105 & 100 \\
\hline
\end{tabular}

4.2 Groups based on outcome

\begin{tabular}{|c|c|c|c|}
\hline Outcome & GOS & Number of patients & Percentage \% \\
\hline Good & $4-5$ & 94 & 89.5 \\
\hline Poor & $1-3$ & 11 & 10.5 \\
\hline \multicolumn{2}{|c|}{ Total } & 105 & 100 \\
\hline
\end{tabular}

\subsection{GCS and GOS Cross-tabulation}

\begin{tabular}{|c|c|c|c|}
\hline \multirow{2}{*}{$\begin{array}{c}\text { Groups Based on } \\
\text { Risk Category }\end{array}$} & \multicolumn{2}{|c|}{ Groups Based on Outcome Category } & \multirow{2}{*}{ Total } \\
\cline { 2 - 3 } & Good & Poor & \\
\hline \multirow{2}{*}{ High risk } & 17 & 6 & 23 \\
& $(73.9 \%)$ & $(26.1 \%)$ & $(100 \%)$ \\
\hline Non-high risk & 77 & 5 & 82 \\
& $(93.9 \%)$ & $(6.1 \%)$ & $(100 \%)$ \\
\hline Total & 94 & 11 & 105 \\
& $(89.5 \%)$ & $(10.5 \%)$ & $(100 \%)$ \\
\hline
\end{tabular}

\subsection{Relationship of GOS with GCS}

\begin{tabular}{|c|c|c|}
\hline Parameters & GOS & GCS \\
\hline Correlation Coefficient & 1.00 & 0.341 \\
\hline$p$-value & \multicolumn{2}{|c|}{$<0.001$} \\
\hline
\end{tabular}


4.5 Relationship of GCS with INR

\begin{tabular}{|c|c|c|}
\hline Parameters & GCS & INR Value \\
\hline Correlation Coefficient & 1.00 & -0.304 \\
\hline$p$-value & \multicolumn{2}{|c|}{0.002} \\
\hline
\end{tabular}

4.6 Relationship of GOS with INR

\begin{tabular}{|c|c|c|}
\hline Parameters & GOS & INR Value \\
\hline Correlation Coefficient & 1.00 & -0.191 \\
\hline$p$-value & \multicolumn{2}{|c|}{0.052} \\
\hline
\end{tabular}

4.7 Median values of INR of Non-high risk and High risk groups

\begin{tabular}{|c|c|c|c|}
\hline \multirow{2}{*}{ Parameter } & \multicolumn{2}{|c|}{ Median (Inter-Quartile Range) } & \multirow{2}{*}{$\boldsymbol{p}$-value } \\
\cline { 2 - 3 } & (Non-high risk group) & (High risk group) & \\
\hline INR value & $1.03(0.99,1.10)$ & $1.10(1.03,1.13)$ & 0.016 \\
\hline
\end{tabular}

4.8 Median values of INR of Good and Poor outcome groups

\begin{tabular}{|c|c|c|c|}
\hline \multirow{2}{*}{ Parameter } & \multicolumn{2}{|c|}{ Median (Inter-Quartile Range) } & \multirow{2}{*}{$\boldsymbol{p}$-value } \\
\cline { 2 - 3 } & (Good outcome) & (Poor outcome) & \\
\hline INR value & $1.04(0.99,1.10)$ & $1.09(1.02,1.13)$ & 0.023 \\
\hline
\end{tabular}

4.9 ROC curve to find the cut-off value of INR for predicting good outcome

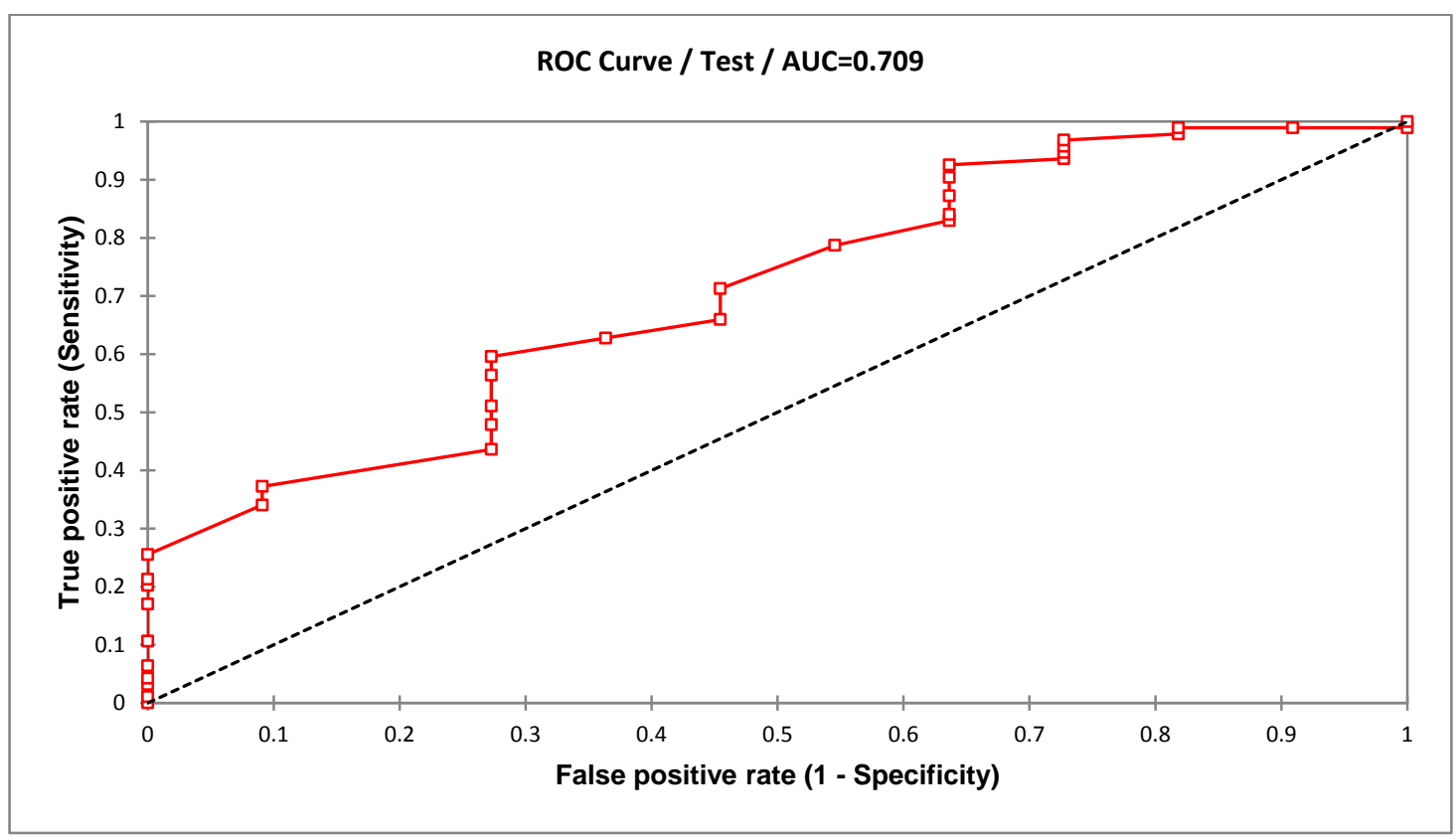




\begin{tabular}{|c|c|}
\hline Parameters & Values \\
\hline Area under the curve & 0.709 \\
\hline Standard error & 0.081 \\
\hline $\begin{array}{c}\text { 95\% asymptomatic } \\
\text { confidence interval }\end{array}$ & $0.550-0.869$ \\
\hline p-value & 0.010 \\
\hline
\end{tabular}

\section{Data Interpretation}

The 105 patients have been separated into groups based on risk and outcome. 94 patients (89.5\%) had good outcome and 11 patients (10.5\%) had poor outcome. In terms of risk, 82 patients (78.1\%) have been classified as non-high risk and 23 patients $(21.9 \%)$ as high risk.

GOS and GCS showed a positive relationship; 0.341 with p-value of $<0.001$. Only $6.1 \%$ of non-high risk group patient had poor outcome as compared to $26.1 \%$ of high risk group. This implies that the lower the GCS score, the poorer the outcome.

GCS and INR showed a negative relationship; -0.304 with p-value of 0.002 . The median value of INR in non-high risk and high risk groups is 1.03 and 1.10 respectively with $p$-value of 0.016 . High risk group of patients had higher INR values. This supports the fact that the traumatic brain injury is associated with a hypocoagulable state.

INR and GOS showed a negative relationship too; -0.191 with p-value of 0.052 . The median values of INR in good and poor outcome are 1.04 and 1.09 respectively with p-value of 0.016 . Thus, a lower value of INR is likely to be associated better outcome of patients.

Using ROC curve, the cut-off value of INR value is 1.06. Values lesser than 1.06 are likely to be associated with good prognosis. The sensitivity is $59.6 \%$, specificity is $72.7 \%$, positive predictive value is $94.9 \%$, and negative predictive value is $17.4 \%$ and accuracy of $61.0 \%$.

Patients with INR value of 1.06 or lesser are likely to have good prognosis as indicated by the high positive predictive value. However, since the negative predictability of this cut-off is low. The converse, whereby INR more than 1.06 is associated with poor prognosis is yet to be justified. This is due to lesser data size of patients with high risk and poor outcome.

\section{Discussions}

GCS is a widely accepted prognostic tool in traumatic brain injury. Patients with lower GCS have higher risk of succumbing to poor outcome. A correct risk assessment was essential in this research to produce reliable relationship between INR with GCS and GOS.

Hence, we studied the relationship between GOS and GCS. The correct application of GCS was confirmed in this study by the positive relationship of GOS and GCS, whereby high risk group patients tend to succumb to poor 
outcome. The relationship between INR and GCS was studied to learn the association of hypocoagulability with the conscious level of patients. The statistically significant negative correlation of INR with GCS showed that patients with lower conscious level may be affected with hypocoagulability. This is further proven as the median INR value of high risk group is higher than non-high risk groups.

The relationship between INR and GOS was studied to note the association of hypocoagulability with the outcome, hence to assess the prognostic predictability of INR. The statistically significant negative correlation of INR with GOS and lower median INR value in patients with good outcome show that patients with initial less-deranged coagulopathy state are likely to progress better.

The cut-off value; 1.06 which is lower than some studies where the cut-off value is $1.50_{(5,8)}$ The other studies only considered fatal cases thereby determining INR as a predictor of mortality. However, our study included both the mortality and morbidity aspects and widening the range of patients considered as poor prognosis and narrowing the patients considered as good prognosis. Both these aspects have been considered in determining the prognosis as they reflect better on the quality of life of patient $\mathrm{s}$ upon discharge. Besides that, the other studies included all trauma cases meanwhile ours only focused on isolated TBI. A lower cut-off value of INR in our study shows hypocoagulability plays a more significant role in TBI.

Based on this result, one may expect patients to improve significantly if INR value is lesser or equal to 1.06. Values higher than 1.06 deserves a caution on the possibility of bad prognosis but it neither necessitates prophylactic antifibrinolytic agents nor to prolong any neurosurgical intervention, provided it is within the normal range ${ }_{(10)}$ Coagulopathy in TBI is usually transient and is variable over its course in the spectrum of TBI. Hence, clinical correlation plays a more significant role before deciding further interventions.

\section{Conclusion}

INR of 1.06 or lesser predicts good prognosis in isolated TBI patients. However, further studies with larger sample size are needed to validate this cut-off value.

\section{Declarations}

\section{Source of Funding}

This research did not receive any specific grant from funding agencies in the public, commercial, or non-profit sectors.

\section{Competing Interests Statement}

The authors declare no competing financial, professional and personal interests.

\section{Ethical Approval}

All experiments were carried out as per institutional guidelines.

\section{Consent to participate}

Not Applicable 


\section{Consent for publication}

We declare that we consented for the publication of this research work.

\section{Availability of data and material}

Authors are willing to share data and material according to the relevant needs.

\section{References}

[1] Gururaj G. Epidemiology of traumatic Brain Injuries: Indian scenario. Neurol Res. 2002 Jan; 24(1):24-8.

[2] Percival H Pangilinan, Brian M Kelly, Joseph E Hornyak, Francisco Talavera, Moichael T, Andary, et all. Classification and complications of Traumatic Brain Injury. Medscape.

[3] Zhang J1, Jiang R, Liu L, Watkins T, Zhang F, Dong JF. Traumatic brain injury-associated coagulopathy. J Neurotrauma. 2012 Nov 20;29(17):2597-605. doi: 10.1089/neu.2012.2348. Epub 2012 Oct 31.

[4] Olson JD, Kaufman HH, Moake J, et al. The incidence and significance of hemostatic abnormalities in patients with head injuries. Neurosurgery 24:825-832, 1989.

[5] G Fuller, H Pattani, A Chalmers, D Sperry, P Yeoman. Coagulopathy predicts poor outcome in traumatic brain injury. Critical Care 2008, 12(Suppl 2):P126 (doi: 10.1186/cc6347).

[6] Jia Liu, Heng-Li Tian. Relationship between trauma-induced coagulopathy and progressive hemorrhagic injury in patients with traumatic brain injury. Chinese Journal of Traumatology 19 (2016) 172e175.

[7] Tian Y, Salsbery B, Wang M, Yuan H, Yang J, Zhao Z, et all.Brain-derived microparticles induce systemic coagulation in a murine model of traumatic brain injury, Blood. 2015 Jan 27.

[8] Jennett B, Bond M. Assessment of outcome after severe brain damage. Lancet. 1975 1(7905):480-4. Medline.

[9] Teasdale, G., (1974). Assessment of coma and impaired consciousness. The Lancet, 304(7872), 81-84.

[10] Ankur Verma, Tamarish Kole. International Normalised Ratio as predictor of mortality in trauma patients in India. World J Emerg Med, Vol 5, No 3, 2014. 